\title{
Variación espacial en número de especies, abundancia y diversidad de peces en las Bahías de Huatulco, Oaxaca, México
}

\author{
Luis Gibran Juárez-Hernández ${ }^{1,2}$ \& Margarito Tapia-García ${ }^{2}$ \\ 1. Centro Universitario CIFE. Calle Tabachin No. 514, Col. Bellavista. C. P. 62140, Cuernavaca, Morelos, México; \\ gibbjuarez@gmail.com \\ 2. Departamento de Hidrobiología. Universidad Autónoma Metropolitana. Unidad Iztapalapa. Av. San Rafael Atlixco \\ No.186, Col. Vicentina. C.P. 09340. Ciudad de México, México; mtg@xanum.uam.mx
}

Recibido 06-I-2017. Corregido 06-VII-2017. Aceptado 08-VIII-2017.

\begin{abstract}
Spatial variation in species number, abundance and diversity of fish in Huatulco Bays, Oaxaca, Mexico. Coral reef fish may adapt to their habitat characteristics, and the particular system intrinsic factors, may cause different fish compositions and structures. The present study aimed to determine the variation in number of species, abundance and diversity of fish communities in Huatulco bays of San Agustin, Riscalillo, Chachacual, Cacaluta, Maguey, Organo, Violín and La Entrega, from Oaxaca, Mexico. Fish communities were characterized between January 2009 and December 2010, with the use of visual census techniques. A total of 351 transects $(10 \times 2.5 \mathrm{~m})$ were made, and for each one we analyzed the ichthyofauna species number, abundance and diversity. The analysis showed that for all variables, the highest values corresponded to Maguey, La Entrega, Órgano and Violín bays, while the lowest values corresponded to the San Agustín, Riscalillo, Chachacual and Cacaluta bays. The study identified significant differences in diversity (Kruskal-Wallis $=18.71, \mathrm{P}=0.0091$ ), and in the composition and structure of fish communities in the bays (ANOSIM=0.278, $\mathrm{P}=0.001)$. We observed a relationship between the number of fish species and number of coral species (Spearman $=0.591, \mathrm{P}<0.05$ ), while no relationship was found between the coral reef area and the number of fish species or their abundances. The number of coral species and the patchy arrangement of the coral cover created habitats that are structurally more complex, which affects the number of fish species. In areas with developed tourist infrastructure, recreational aquatic activities were found to affect the ichthyofauna behavior, abundance and diversity. The new information of this study may contribute to create and improve management, conservation and protection strategies for this important area as a whole interconnected complex. Rev. Biol. Trop. 65 (4): 1407-1418. Epub 2017 December 01.
\end{abstract}

Key words: ichthyofauna, diversity, corals, tourism, conservation, Mexican Pacific.

Los peces estan adaptados a diferentes características del hábitat arrecifal (Choat, \& Bellwood, 1991), por lo tanto, las diferencias en la composición y estructura íctica pueden ser resultado de factores intrínsecos de cada sistema (Almany, 2004; Álvarez- Filip, 2004). A este respecto, el área coralina, riqueza de especies coralinas y proporción de sustratos, han sido considerados como atributos que influencian la abundancia y diversidad íctica (Chabanet, Ralambondrainy, Amanieu, Fauré, \& Galzin 1997; Friedlander, \& Parrish, 1998; Acosta, \& Robertson, 2002; ÁlvarezFilip, Dulvy, Côté, Watkinson, \& Gill, 2011).
Aunado a estos factores, se ha demostrado que la composición y estructura íctica también puede ser influenciada por la cercanía a núcleos urbanos y prácticas turísticas (Milazzo, Anastasi, \& Willis, 2006; Di Franco, Baiata, \& Milazzo, 2013).

La ictiofauna de Bahías de Huatulco ha sido considerada como una de las de mayor riqueza íctica dentro del Pacífico mexicano (LópezPérez et al., 2010; 2014; Juárez-Hernández, 2014). Para este conjunto de bahías, se han mencionado amplias variaciones en la abundancia y diversidad íctica, así como diferencias significativas en términos de composición 
y estructura (López-Pérez, Calderón-Aguilera, Zepeda-Vilchis, López Pérez-Maldonado, \& López-Ortiz, 2012; Juárez-Hernández, TapiaGarcía, \& Luna-Monsiváis, 2013). Del total de bahías, Cacaluta, San Agustín, La Entrega y Maguey, han sido señaladas como las de mayor diversidad de peces (Ramírez-Gutiérrez, Tapia-García, Ramos-Santiago, \& Ulloa, 2007; López-Pérez et al., 2010; Juárez-Hernández, 2014), pero para las bahías Órgano y Violín, se cuenta con pocos antecedentes de la ictiofauna.

En la zona se desarrollan actividades económicas, principalmente un desarrollo turístico de importancia nacional que ha generado problemas en la calidad ambiental, contaminación y degradación en los ecosistemas (López-Pérez, \& Hernández-Ballesteros, 2004; FonsecaGally, 2010). En 1998, las bahías de Huatulco fueron decretadas como Parque Nacional por el gobierno mexicano, considerando como un criterio de inclusión, los arrecifes de las bahías Cacaluta, Chachacual, Riscalillo y San Agustín (CONANP, 2003). Se destaca que la bahía La Entrega, a pesar de poseer uno de los arrecifes de mayor dimensión de la región y poseer una alta riqueza de especie coralinas (López-Pérez,
\& Hernández-Ballesteros, 2004; Tapia-García, Stender, \& Juárez-Hernández, 2007), no fue considerada dentro de la poligonal del Parque Nacional. Probablemente esta bahía sea una de las más impactadas en su arrecife, determinado por la relativa facilidad de acceso, infraestructura turística y actividades acuáticas. A este respecto, estas actividades también han determinado deterioro en las comunidades coralinas e ícticas de las bahías de San Agustín y Maguey, las cuales se incluyen dentro del parque nacional (Fonseca-Gally, 2010; Ramírez-Gutiérrez, 2010; Juárez-Hernández, 2014). Por lo tanto, el presente estudio tiene como finalidad, determinar la abundancia, número de especies y diversidad de peces de las bahías de Huatulco, con el objetivo de actualizar la información existente y para las bahías de Órgano y Violín, contribuir con el conocimiento de su ictiofauna.

\section{MATERIALES Y MÉTODOS}

En la costa de Oaxaca se localiza el complejo "Bahías de Huatulco" (1540"48" - 1545'36" N \& 96¹4'24” - 9607'13” W) (Fig. 1). La cobertura coralina de la zona

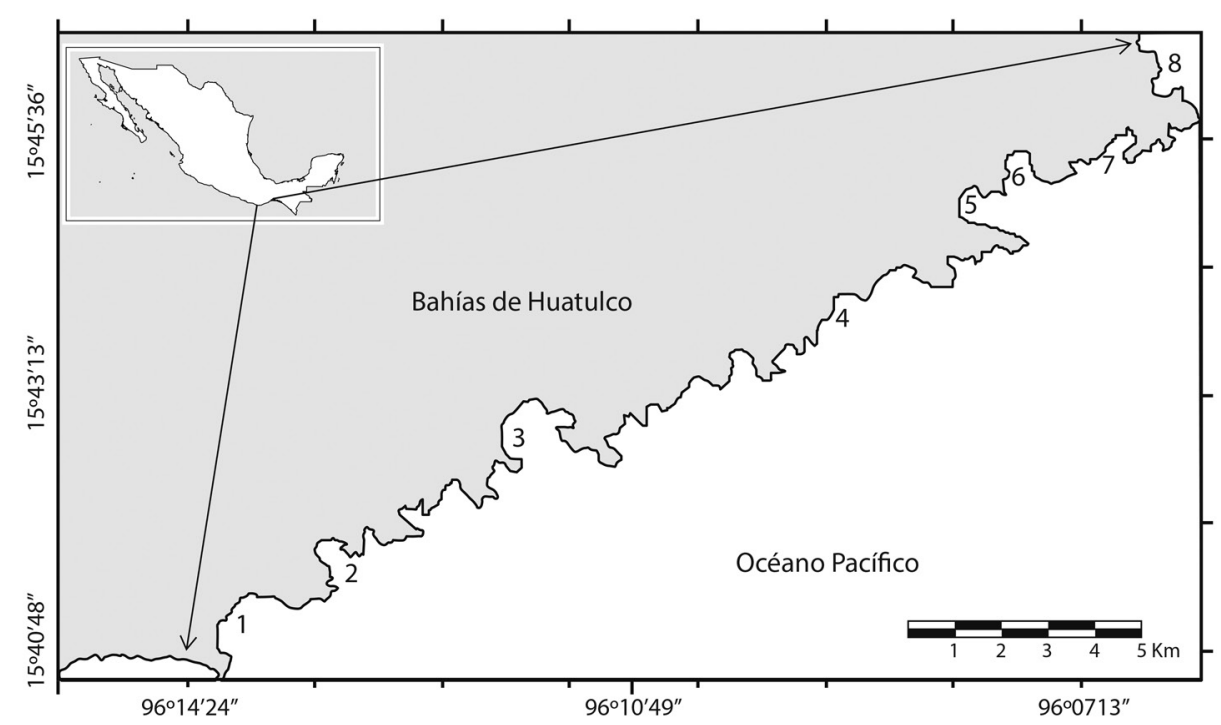

Fig. 1. Área de estudio. Bahías de Huatulco: 1) San Agustín, 2) Riscalillo, 3) Chachacual, 4) Cacaluta, 5) Maguey, 6) Órgano, 7) Violín y 8) La Entrega.

Fig. 1. Location of the study area, Huatulco Bays: 1) San Agustin, 2) Riscalillo, 3) Chachacual, 4) Cacaluta, 5) Maguey, 6) Organo, 7) Violin and 8) La Entrega. 
es de aproximadamente de 45.9 hectáreas, y está compuesta de 17 arrecifes bordeantes y 11 especies de corales hermatípicos (Glynn, \& Leyte-Morales, 1997; López-Pérez, \& Hernández-Ballesteros, 2004; Tapia-García et al., 2007). Este complejo está integrado por once bahías, de las cuales San Agustín, Riscalillo, Chachacual, Cacalutla, Maguey, Órgano y Violín se suscriben dentro del Parque Nacional Huatulco.

En las localidades mencionadas, se realizó un muestreo de prospección desde inicios de enero 2009, con el objetivo de definir y registrar la ubicación geográfica de cada punto de muestreo mediante el uso de un geoposicionador satelital (GPS). Posterior a este procedimiento, se diseñó un método para la obtención de la representación de sustratos por punto de muestreo. Este método consistió en colocar un transecto horizontal de 10 metros de longitud y otro transecto de la misma longitud, colocado a la mitad del primero (i. e. a los cinco metros), con el objetivo de crear cuatro cuadrantes de $25 \mathrm{~m}^{2}$ cada uno. En cada cuadrante el observador estimaba visualmente la proporción de sustratos, y con la suma de cada cuadrante se determinó la representación de cada sustrato (coral, roca, arena y escombro) por punto de muestreo. Obtenida esta información, se determinó la representación porcentual de cada sustrato por localidad. El área coralina y número de especies coralinas por localidad, se obtuvo de la consulta de los trabajos de Glynn y Leyte-Morales (1997), CONANP (2003), López-Pérez y Hernández-Ballesteros (2004), Alejandre-Samaniego (2010), Fonseca-Gally (2010), y Tapia-García et al. (2007).

La caracterización de la comunidad de peces se realizó a través de la técnica de censos visuales, mediante transectos (Sale \& Douglas, 1981), de $10 \mathrm{~m}$ de longitud y 2.5 de ancho, a una profundidad mínima de un metro y una máxima de $10 \mathrm{~m}$. La longitud del transecto fue elegida para poder realizar comparaciones con los antecedentes existentes del área de estudio, así como por las dimensiones de los parches coralinos de Maguey y Órgano. Se efectuó un total de 351 transectos, de los cuales 51 se realizaron en San Agustín, 39 en Riscalillo, 30 en Chachacual, 27 en Cacaluta, 72 en Maguey, 22 en Órgano, 46 en Violín y 66 en La Entrega, entre enero 2009 a diciembre 2010. Estos transectos se realizaron en los mismos puntos donde se efectuó la estimación visual de sustratos. En cada transecto, los censos visuales fueron realizados por dos buzos, uno de cada lado del transecto a una distancia de $2.5 \mathrm{~m}$ entre transecto y buzo, y aproximadamente, se consideró un campo de visión de otros $2.5 \mathrm{~m}$ de distancia del lado opuesto del transecto, cubriendo un total de $100 \mathrm{~m}^{2}$. Se realizó el recorrido hacia el otro extremo del transecto y de regreso hacia el punto de origen, registrando las especies en un intervalo de cinco minutos. El segundo recorrido tuvo el objetivo de registrar únicamente a las especies de hábitos crípticos. En su totalidad, los transectos fueron realizados por las mismas personas, en los mismos sitios y empleando el mismo método de muestreo. Las especies de peces censadas fueron identificadas de acuerdo con los trabajos de Allen y Robertson (1998) y Amezcua-Linares (2009).

En cada bahía se determinó el número de especies, abundancia y diversidad (H) (Shannon \& Wiener, 1963). El análisis de estos descriptores se efectuó mediante un análisis de varianza de una vía o la prueba de Kruskal-Wallis, con su respectiva prueba de comparación múltiple (Tukey, Dunn). La pertinencia de aplicación de las pruebas mencionadas fue determinada por el cumplimento de los supuestos de normalidad y homocedasticidad. La evaluación del grado de similitud de la ictiofauna entre localidades, se realizó mediante el índice de Bray-Curtis (Clarke \& Warwick, 1994). La representación gráfica de la similitud se efectuó a través de un análisis de clasificación (dendrograma), con el algoritmo de ligamiento promedio no-ponderado (UPGMA), y una ordenación (escalamiento multidimensional no métrico). Se realizó un análisis de similitud (ANOSIM) (Clarke, 1993) para la identificación de diferencias significativas en términos de composición y abundancia íctica entre localidades. Estos análisis se realizaron con el paquete estadístico PRIMER v6 (Clarke \& Gorley, 2006). 
Finalmente, mediante el coeficiente de correlación de Spearman se evaluó la relación del número de especies, abundancia y diversidad de peces respecto al número de transectos, área coralina, número de especies coralinas, proporción del sustrato coralino y rocoso.

\section{RESULTADOS}

La caracterización de sustratos reveló que San Agustín, Cacaluta, Violín y La Entrega, presentaron la mayor proporción del sustrato coralino, mientras que Órgano y Maguey, registraron la mayor proporción del sustrato rocoso (Cuadro 1). De acuerdo con la consulta de antecedentes, San Agustín, Riscalillo y La Entrega poseen un área coralina mayor a dos hectáreas, mientras que el mayor número de especies coralinas fue característico de Violín, Maguey y Órgano (Cuadro 1).

En las bahías estudiadas se identificaron 23374 peces pertenecientes a 88 especies (Cuadro 2), y 37 familias. Las familias
Labridae, Pomacentridae y Carangidae fueron las de mayor representación. Las especies con la mayor abundancia relativa fueron Thalassoma lucasanum, Stegastes acapulcoensis, Chromis atrilobata y Microspathodon dorsalis (Cuadro 2). En las bahías de San Agustín, Riscalillo, Chachacual y Cacaluta, estas cuatro especies presentaron la mayor abundancia relativa. En Maguey, Órgano, Violín y La Entrega, además de estas cuatro especies, se incluye a Selar crumenophthalmus, Harengula thrissina y Haemulon maculicauda como las de mayor abundancia. La mayor abundancia, diversidad y el mayor número de especies, fueron característicos de las bahías Maguey y La Entrega, mientras que los valores mínimos se registraron de las bahías Cacaluta, Chacual y San Agustín, respectivamente (Cuadro 1). La diversidad presentó diferencias significativas entre localidades (Kruskal-Wallis $=18.71, \mathrm{P}=0.0091$ ). En este orden, la prueba de comparación múltiple de Dunn indicó que esta diferenciación fue determinada por San Agustín respecto

\section{CUADRO 1}

Número de transectos, número de especies, abundancia, diversidad (media \pm desviación estándar) de las comunidades de peces, área coralina, número de especies coralinas y tipo de sustrato por bahía

TABLE 1

Transects number, species number, abundance, diversity (mean \pm standard deviation) of fish communities, coral areas, number of coral species and type of substrates by each bay

\begin{tabular}{|c|c|c|c|c|c|c|c|c|c|c|}
\hline Bahía & $\begin{array}{l}\text { Núm. de } \\
\text { transectos }\end{array}$ & $\begin{array}{c}\text { Núm. de especies } \\
\text { de peces }\end{array}$ & $\begin{array}{l}\text { Abundancia } \\
\text { (Individuos) }\end{array}$ & $\begin{array}{c}\text { Diversidad } \\
(\mathrm{H})\end{array}$ & $\begin{array}{c}\text { Área } \\
\text { coralina (ha) }\end{array}$ & $\begin{array}{l}\text { Núm. de especies } \\
\text { coralinas }\end{array}$ & $\begin{array}{l}\text { Coral } \\
(\%)\end{array}$ & $\begin{array}{l}\text { Roca } \\
(\%)\end{array}$ & $\begin{array}{c}\text { Arena } \\
(\%)\end{array}$ & $\begin{array}{c}\text { Escombro } \\
(\%)\end{array}$ \\
\hline San Agustín & 51 & 46 & 3495 & $\begin{array}{c}1.24 \\
( \pm 0.391)\end{array}$ & 2.7 & 6 & 65 & 20 & 7 & 8 \\
\hline Riscalillo & 39 & 48 & 2339 & $\begin{array}{c}1.32 \\
( \pm 0.438)\end{array}$ & 2.5 & 7 & 45 & 5 & 30 & 20 \\
\hline Chachacual & 30 & 44 & 1781 & $\begin{array}{c}1.44 \\
( \pm 0.395)\end{array}$ & 0.5 & 6 & 30 & 20 & 40 & 10 \\
\hline Cacaluta & 27 & 49 & 1609 & $\begin{array}{c}1.45 \\
( \pm 0.371)\end{array}$ & 1.3 & 7 & 65 & 20 & 15 & 0 \\
\hline Maguey & 72 & 69 & 4387 & $\begin{array}{c}1.52 \\
( \pm 0.501)\end{array}$ & 0.44 & 8 & 35 & 40 & 25 & 0 \\
\hline Órgano & 22 & 56 & 1755 & $\begin{array}{c}1.47 \\
( \pm 0.565)\end{array}$ & 0.7 & 8 & 30 & 45 & 25 & 0 \\
\hline Violín & 46 & 50 & 3686 & $\begin{array}{c}1.46 \\
( \pm 0.385)\end{array}$ & 0.57 & 9 & 65 & 25 & 10 & 0 \\
\hline La Entrega & 66 & 60 & 4322 & $\begin{array}{c}1.49 \\
( \pm 0.362)\end{array}$ & 2.35 & 7 & 60 & 10 & 15 & 10 \\
\hline
\end{tabular}


CUADRO 2

Abundancia relativa de las especies de peces de Bahías de Huatulco

TABLE 2

Relative abundance of fish species of Huatulco Bays

\begin{tabular}{|c|c|c|c|c|c|}
\hline Especie & Total & Especie & Total & Especie & Total \\
\hline Thalassoma lucasanum & 27.35 & Scarus compressus & 0.34 & Sphoeroides lobatus & 0.02 \\
\hline Stegastes acapulcoensis & 20.84 & Abudefduf concolor & 0.33 & Elagatis bipinnulata & 0.02 \\
\hline Chromis atrilobata & 9.17 & Holacanthus passer & 0.33 & Synodus lacertinus & 0.02 \\
\hline Microspathodon dorsalis & 5.77 & Chaetodon humeralis & 0.30 & Eucinostomus currani & 0.02 \\
\hline Selar crumenophthalmus & 5.63 & Cephalopholis panamensis & 0.25 & Plagiotremus azaleus & 0.02 \\
\hline Haemulon maculicauda & 4.12 & Stegastes rectifraenum & 0.22 & Urobatis concentricus & 0.01 \\
\hline Prionurus punctatus & 3.74 & Hemicaranx leucurus & 0.20 & Urotrygon rogersi & 0.01 \\
\hline Abudefduf troschelii & 2.63 & Sargocentron suborbitalis & 0.20 & Arothron hispidus & 0.01 \\
\hline Ophioblennius steindachneri & 1.47 & Epinephelus labriformis & 0.17 & Bothus constellatus & 0.01 \\
\hline Haemulon sexfasciatum & 1.34 & Cirrhitus rivulatus & 0.17 & Aetobatus narinari & 0.01 \\
\hline Bodianus diplotaenia & 1.24 & Haemulon steindachneri & 0.16 & Myripristis leiognathus & 0.01 \\
\hline Halichoeres dispilus & 1.07 & Sufflamen verres & 0.15 & Gnathanodon speciosus & 0.01 \\
\hline Caranx caballus & 1.02 & Caranx caninus & 0.15 & Lutjanus colorado & 0.01 \\
\hline Apogon pacificus & 0.95 & Prionurus laticlavius & 0.13 & Novaculichthys taeniourus & 0.01 \\
\hline Harengula thrissina & 0.85 & Apogon retrosella & 0.12 & Zanclus cornutus & 0.01 \\
\hline Halichoeres notospilus & 0.85 & Balistes polylepis & 0.10 & Gymnura marmorata & 0.009 \\
\hline Stegastes flavilatus & 0.82 & Acanthurus xanthopterus & 0.10 & Gymnomuraena zebra & 0.009 \\
\hline Fistularia commersonii & 0.71 & Mulloidichthys dentatus & 0.09 & Quassiremus nothochir & 0.009 \\
\hline Lutjanus argentiventris & 0.66 & Scarus ghobban & 0.09 & Hoplopagrus guentherii & 0.009 \\
\hline Halichoeres nicholsi & 0.56 & Diodon hystrix & 0.09 & Aluterus scriptus & 0.009 \\
\hline Cirrhitichthys oxycephalus & 0.54 & Trachinotus rhodopus & 0.08 & Dasyatis longa & 0.004 \\
\hline Kyphosus elegans & 0.50 & Gerres cinereus & 0.08 & Myrichthys tigrinus & 0.004 \\
\hline Mugil curema & 0.48 & Haemulon flaviguttatum & 0.07 & Epinephelus niphobles & 0.004 \\
\hline Hemiramphus saltator & 0.46 & Diodon holocanthus & 0.06 & Pareques viola & 0.004 \\
\hline Platybelone argalus pterura & 0.46 & Haemulon scudderii & 0.06 & Acanthurus nigricans & 0.004 \\
\hline Microspathodon bairdii & 0.44 & Iniistius pavo & 0.05 & Cantherhines dumerilii & 0.004 \\
\hline Arothron meleagris & 0.41 & Caranx vinctus & 0.04 & Ostracion meleagris & 0.004 \\
\hline Johnrandallia nigrirostris & 0.40 & Melichthys niger & 0.03 & Canthigaster punctatissima & 0.004 \\
\hline Kyphosus analogus & 0.38 & Muraena lentiginosa & 0.03 & & \\
\hline Halichoeres chierchiae & 0.35 & Lutjanus novemfasciatus & 0.03 & & \\
\hline
\end{tabular}

a Maguey, La Entrega, Órgano y Violín, ya que los valores del estadístico de contraste $(Z)$ fueron mayores a 1.96 .

La clasificación de las comunidades de peces reveló la formación de dos grupos de mayor similitud, siendo el primero integrado por la ictiofauna de las bahías Riscalillo, Cacaluta y Chachacual, mientras que el segundo grupo fue conformado por las bahías Maguey, La Entrega y Violín (Fig. 2). La ictiofauna de Órgano presentó una baja similitud. El análisis de similitud reveló diferencias en el patrón multivariado íctico entre localidades (ANOSIM= $0.278 ; \mathrm{P}=0.001)$. A este respecto, el análisis por pares indicó que la comunidad íctica de San Agustín presentó un mayor número de diferencias respecto al resto de localidades (Cuadro 3 ).

Se encontró relación entre la abundancia de peces y el número de transectos (Spearman $=0.857, \mathrm{P}<0.05$ ), no siendo así con el número de especies de peces (Spearman $=0.28$, $\mathrm{P}>0.05$ ). Es relevante indicar que el número 


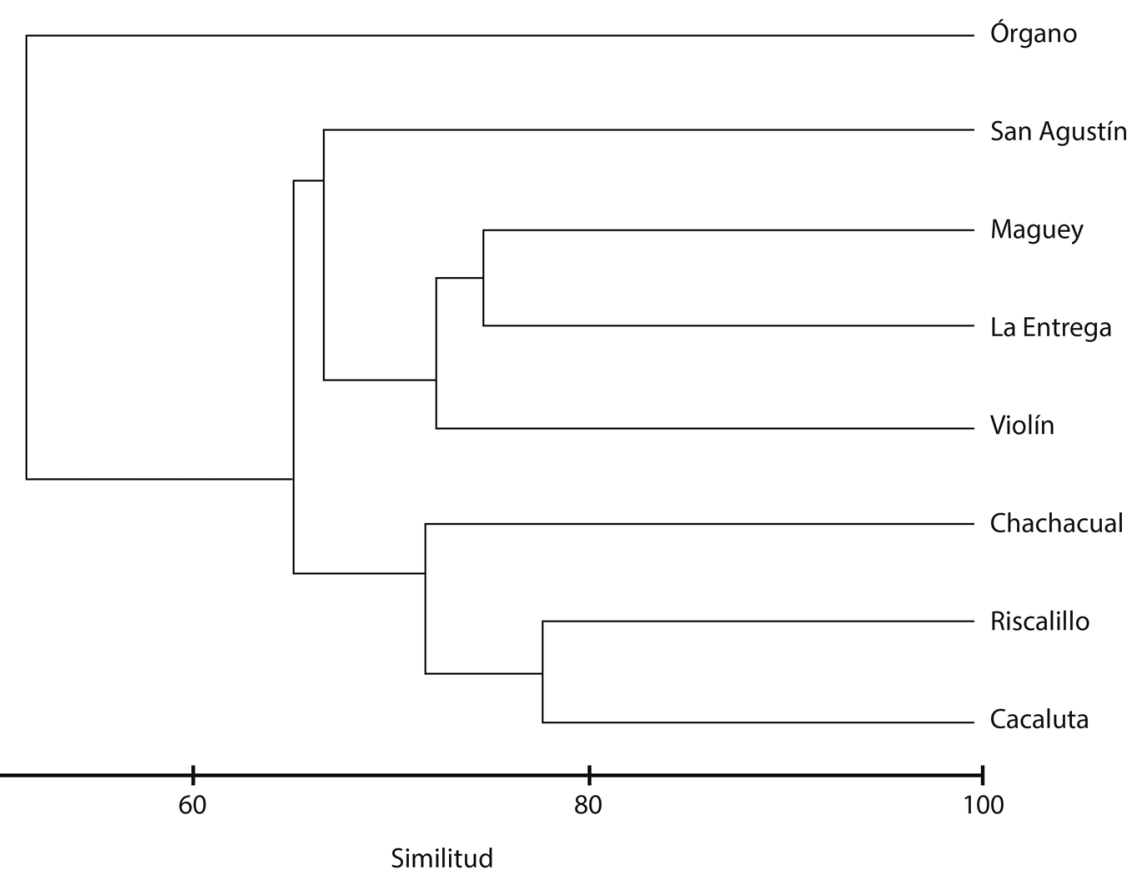

Fig. 2. Diagrama de similitud de las comunidades de peces de las Bahías de Huatulco.

Fig. 2. Similarity diagram of fish communities of Huatulco Bays.

de especies de peces se relacionó con el número de especies coralinas (Spearman $=0.591$, $\mathrm{P}<0.05)$. No se encontraron relaciones significativas del número de especies, abundancia y diversidad de peces respecto al área coralina, proporción de sustrato coralino, rocoso y arenoso $(\mathrm{P}>0.05)$.

\section{DISCUSIÓN}

El número de especies de peces identificadas en el presente estudio es similar al reportado por López-Pérez et al. (2010). Como se mencionó, T. lucasanum, S. acapulcoensis y Ch. atrilobata, presentaron la mayor abundancia relativa, de manera similar con lo previamente reportado para la zona de estudio (RamírezGutiérrez et al., 2007; López-Pérez et al., 2012; Juárez-Hernández et al., 2013). S. crumenophthalmus y $H$. thrissina han sido consideradas como visitantes cíclicos en Bahías de Huatulco (Ramírez-Gutiérrez et al., 2007, López-Pérez et al., 2012) y su presencia se encuentra asociada a cuestiones alimentarias, ya que suelen acercarse a la zona costera en los meses donde el efecto de los vientos "Tehuanos" transportan agua rica en nutrientes de esta forma elevando la producción primaria en las bahías (MonrealGómez, \& Salas de León, 1998).

El análisis del número de especies, abundancia y diversidad de la ictiofauna reveló una tendencia general de los valores máximos y mínimos entre localidades, ya que los valores máximos fueron característicos de las bahías Maguey, La Entrega, Órgano y Violín; mientras que los valores mínimos fueron propios de San Agustín, Riscalillo, Chachacual y Cacaluta. La tendencia observada fue consistente con la clasificación de bahías obtenida, con una baja similitud de Maguey, Órgano, Violín y La Entrega respecto a San Agustín, Riscalillo, Chachacual y Cacaluta. El análisis de similitud y análisis de varianza confirmaron la existencia de diferencias significativas en la composición, abundancia y diversidad entre las localidades estudiadas. De acuerdo a la prueba de comparación múltiple y prueba por pares de los análisis 
CUADRO 3

Resultados de la prueba por pares del análisis de similitud (ANOSIM)

TABLE 3

Results of pairwise test of ANOSIM

\begin{tabular}{|c|c|c|}
\hline \multicolumn{2}{|c|}{ Grupos } & \multirow{2}{*}{$\begin{array}{c}\mathrm{R} \\
0.409 *\end{array}$} \\
\hline San Agustín & Riscalillo & \\
\hline San Agustín & Chachacual & $0.5^{*}$ \\
\hline San Agustín & Cacaluta & $0.233^{*}$ \\
\hline San Agustín & Maguey & 0.477 * \\
\hline San Agustín & Órgano & $0.761^{*}$ \\
\hline San Agustín & Violín & $0.365^{*}$ \\
\hline San Agustín & La Entrega & $0.24 *$ \\
\hline Riscalillo & Chachacual & $0.328 *$ \\
\hline Riscalillo & Cacaluta & $0.211^{*}$ \\
\hline Riscalillo & Maguey & 0.15 \\
\hline Riscalillo & Órgano & $0.301^{*}$ \\
\hline Riscalillo & Violín & $0.282 *$ \\
\hline Riscalillo & La Entrega & 0.121 \\
\hline Chachacual & Cacaluta & $0.169 *$ \\
\hline Chachacual & Maguey & $0.271^{*}$ \\
\hline Chachacual & Órgano & 0.427 * \\
\hline Chachacual & Violín & $0.415^{*}$ \\
\hline Chachacual & La Entrega & $0.253^{*}$ \\
\hline Cacaluta & Maguey & 0.237 \\
\hline Cacaluta & Órgano & $0.181^{*}$ \\
\hline Cacaluta & Violín & 0.347 * \\
\hline Cacaluta & La Entrega & $0.311^{*}$ \\
\hline Maguey & Órgano & 0.127 \\
\hline Maguey & Violín & $0.335^{*}$ \\
\hline Maguey & La Entrega & 0.072 \\
\hline Órgano & Violín & $0.491 *$ \\
\hline Órgano & La Entrega & $0.456^{*}$ \\
\hline Violín & La Entrega & $0.236^{*}$ \\
\hline
\end{tabular}

Las diferencias son significativas si $\mathrm{P}<0.05\left(^{*}\right)$.

The differences are significant if $\mathrm{P}<0.05\left(^{*}\right)$.

referidos, la ictiofauna de San Agustín se diferenció del resto de localidades. La tendencia observada y diferenciación de la ictiofauna de San Agustín, coincide con los propuesto por López-Pérez et al. (2012). Probablemente, la diferenciación de las comunidades ícticas sea consistente con la diferenciación de las comunidades coralinas de la zona de estudio, la cual es resultado de la variabilidad en el número de especies coralinas y diversidad entre localidades (López-Pérez, \& Hernández-Ballesteros; 2004).

Se ha mencionado que la abundancia y diversidad íctica pudiera estar influenciada principalmente por el área coralina, número de especies coralinas y configuración de la cobertura coralina (Sale \& Dybdahl, 1975; Sale \& Douglas, 1984; Álvarez-Filip et al., 2011; Acosta, \& Robertson, 2002; Gratwicke \& Speight, 2005a). A este respecto, se esperaba que San Agustín, Riscalillo y La Entrega presentaran un mayor número de individuos y especies de peces, resultado de su área coralina, sin embargo, en el presente estudio no se observó esta relación. Lo anterior, probablemente sea determinado por las características del área coralina de estas localidades, ya que en San Agustín y La Entrega, está dispuesta en su mayoría en una placa coralina continua, constituida por pocas especies coralinas (López-Pérez, \& Hernández-Ballesteros, 2004; Fonseca-Gally, 2010) y con escasa complejidad topográfica (Ramírez-Gutiérrez, 2010). En este orden, el mayor número de especies de peces en ambas localidades se registró en áreas donde el sustrato coralino tiene una escasa representación. Arias-González, Núñez-Lara, RodríguezZaragoza y Legendre (2011) indican que el área coralina es relevante en la determinación del número de especies de peces, cuando posee una alta cobertura de coral vivo y una significativa complejidad topográfica. Esta relación se fundamenta en que la cobertura de coral vivo provee sitios de forrajeo, desove y anidación, así como abundantes refugios para los peces (Munday, 2000).

La complejidad topográfica es influenciada por el número de especies coralinas (Álvarez-Filip et al., 2011) y disposición en parches de la cobertura coralina (Acosta, \& Robertson, 2002). Este argumento es consistente con los resultados de este estudio, ya que existió relación del número de especies de peces con el número de especies coralinas. Como se mencionó, la bahía Maguey presentó el mayor número de especies de peces y diversidad, caracterizándose por presentar un alto número de especies coralinas. En este orden, 
Álvarez-Filip et al. (2011) indican que sitios con cinco o menos especies coralinas, tienden a ser relativamente planos, mientras que sitios con ocho a 13 especies de coral, presentan mayor complejidad topográfica, resultado de la forma y crecimiento diferencial de cada especie, lo que ofrece mayor disponibilidad de refugios, microhábitats y recursos para la ictiofauna (Chabanet et al., 1997; Nanami \& Nishihira, 2002). Aunado al número de especies coralinas, una característica relevante de esta localidad, así como de Órgano, es que el área coralina está constituida por pequeños parches, con una alternancia con el sustrato rocoso o arenoso, lo que genera áreas tridimensionalmente complejas, con un contraste en el relieve vertical, por lo tanto con un mayor número de áreas de refugio y alimento para los peces (Acosta, \& Robertson, 2002; Charbonnel, Serre, Ruitton, Harmelin, \& Jensen, 2002; Gratwicke \& Speight, 2005a, b; Tokeshi, \& Arakaki, 2012).

A pesar de que la cobertura rocosa no mostró relación con los descriptores de la comunidad íctica, se observó una tendencia destacable, la cual consistió en que las localidades con una alta proporción del sustrato rocoso (i. e. Maguey y Órgano), presentaron una alta diversidad y número de especies de peces. Esta relación se fundamenta en que las características geológicas y físiográficas del sustrato rocoso, determinan superficies complejas y rugosas, con un mayor número de microhábitats, refugios y recursos para los peces (Aburto-Oropeza, \& Balart, 2001; Galván-Villa, Arreola-Robles, Ríos-Jara, \& Rodríguez-Zaragoza, 2010; Giakoumi, \& Kokkoris, 2013). En este orden, Gratwicke y Speight (2005a) indican que el sustrato rocoso permite el desarrollo y permanencia de microalgas, macroalgas, corales, esponjas, así como una gran variedad de organismos parcialmente sésiles y sésiles.

Como se indicó antes, la abundancia de peces estuvo influenciada por el número de transectos. En este orden, en las localidades donde se realizó un mayor número de transectos, fue por la cercanía al núcleo poblacional.
En caso contrario, en Cacalutla se efectuó un menor número de transectos, relacionado directamente con condiciones adversas para el arribo, así como el establecimiento de los puntos de muestreo y ejecución del censo por el oleaje. A este respecto, el número de especies y abundancia de peces censados en San Agustín, Cacaluta y La Entrega, presentaron diferencias respecto a los antecedentes (Ramírez-Gutiérrez et al., 2007; López-Pérez et al., 2010; JuárezHernández et al., 2013), probablemente resultado del número de transectos efectuados.

Una contribución relevante del presente estudio, fue proporcionar información referente a la comunidad íctica de las bahías Órgano y Violín. En este orden, Bahía Órgano, presentó un número alto de especies de peces, aun cuando el número de transectos fue menor respecto al resto de localidades. Con respecto a Violín, se señala que el número de especies y diversidad de peces son similares a los descritos por López-Pérez et al. (2012).

En el presente trabajo se observó la influencia de las actividades recreativas acuáticas en el comportamiento de los peces, con implicaciones en su abundancia y número de especies. Maguey y La Entrega se ubican cerca al núcleo poblacional, presentan acceso por tierra y poseen un desarrollo turístico, por lo que la presencia de turistas y las actividades recreativas acuáticas son comunes. En Maguey, particularmente se identificó la adecuación (i. e. no evitan el contacto) de las diferentes especies de peces a la presencia de turistas que desarrollan actividades acuáticas. Probablemente, esta conducta es influenciada por el alimento que ofrecen los prestadores de servicios a los peces en las visitas guiadas. Milazzo et al. (2006), refieren a esta actividad como alimentación recreativa y concluyen que genera cambios en la conducta, abundancia y distribución de la ictiofauna, ya que disminuye "el miedo" instintivo hacia el humano. Probablemente, esta condición influyó en el número de especies y abundancia censadas, ya que durante el censo resultaría de mayor facilidad detectar e identificar a las especies de peces, a diferencia del comportamiento observado 
en otras localidades (Riscalillo, Chachacual, Cacaluta, Órgano y Violín), en las que ante la presencia de los observadores, los peces tendían a escapar. En La Entrega, se observó que el contacto continuo de los bañistas en ambientes arenosos, atrae a un conjunto de especies de peces (Scarus compressus, Balistes polylepis, Halichoeres nicholsi, Diodon hystrix y Mugil curema). Generalmente estas especies se observaron cerca de los bañistas, y posterior al contacto con el sustrato, estos acudían de manera inmediata al sitio de contacto, coincidiendo con lo observado por Di Franco et al. (2013). Estos autores indican que la remoción del sustrato por estas actividades, permite que las presas de estas especies sean más accesibles y determinan la disminución de la desconfianza instintiva de los peces hacia los humanos en zonas donde el turismo es continuo, coincidiendo con los resultados del presente trabajo.

Otra implicación de las actividades acuáticas en las Bahías de Huatulco, se relacionan con el deterioro que ocasionan en las comunidades coralinas. En San Agustín, se ha determinado un alto porcentaje de coral erosionado y muerto en donde se realizan actividades subacuáticas y recreativas intensas, resultado en el daño continuo que bañistas ocasionan al ponerse de pie sobre la placa coralina (Fonseca-Gally, 2010), coincidiendo con lo propuesto por Hawkins y Roberts (1993). El deterioro en la comunidad coralina se ha reflejado en la comunidad íctica, ya que se ha cuantificado una disminución del índice de diversidad (Ramírez-Gutiérrez, 2010). A este respecto, las comunidades coralinas de Bahías de Huatulco están constituidas en su mayoría por el género Pocillopora, siendo de los géneros más susceptibles de morir, dejar de reproducirse sexualmente o blanquearse, por efecto de impactos turísticos (Zakai, Levy, \& Chadwick-Furman, 2000). Dentro de las especies pertenecientes a este género, destaca $P$. damicornis, cuál ha sido considerada altamente frágil y de ramas quebradizas, lo que incrementa su susceptibilidad al daño por actividades humanas. Lo anterior es relevante, ya que esta especie es de las de mayor representación en las comunidades coralinas de la zona de estudio (López-Pérez, Mora-Pérez, \& Leyte-Morales, 2007).

Finalmente, disturbios en la comunidad coralina relacionados con el desarrollo de infraestructura turística y por factores naturales (i. e. variaciones en la temperatura del mar) han sido observados en Bahías de Huatulco. JuárezHernández (2014) refiere que la construcción de andadores turísticos propicio el ingreso de sedimento terrígeno a las comunidades coralinas de las bahías Maguey y Violín, provocando mortalidad coralina, y determinando decrementos en el número de especies, abundancia y diversidad íctica. Por factores naturales, en Riscalillo y Chachacual se ha documentado eventos de mortalidad coralina (López-Pérez et al., 2002; Alejandre-Samaniego, 2010), probablemente lo anterior sea consistente con el número de especies censadas en el presente. Muday (2004) refiere que la disminución de cobertura de coral vivo, cambios la composición de especies, y la subsecuente perdida de complejidad topográfica de la comunidad coralina, determinan el colapso de la diversidad y abundancia de la comunidad íctica.

El número de especies, abundancia y diversidad de peces presentó diferencias en las bahías de Huatulco. Esta diferenciación es influenciada por las características intrínsecas de cada localidad y actividades que en ellas se desarrollan. A este respecto, se recomiendan estudios que evalúen la influencia en la composición y estructura íctica de algunas características no contempladas en el presente, como por ejemplo el estado ecológico de la comunidad coralina, así como la composición y estructura de la comunidad algal. Información actual y especifica de las comunidades icticas y coralinas e incorporación de políticas de regulación de las actividades recreativas acuáticas, permitirá la implementación de estrategias oportunas de conservación y manejo para los recursos naturales de la región.

\section{AGRADECIMIENTOS}

Este trabajo se realizó a través del proyecto de investigación "Diagnóstico ecológico 
de los sistemas acuáticos de México, como base para su gestión ambiental" del departamento de Hidrobiología de la Universidad Autónoma Metropolitana, Unidad Iztapalapa. A las autoridades del Parque Nacional Huatulco, por el apoyo brindado para la autorización y ejecución del presente estudio.

\section{RESUMEN}

Los peces arrecifales están adaptados a diferentes características de su hábitat, por lo tanto, las diferencias en composición y estructura pueden ser resultado de factores intrínsecos de cada sistema. En este trabajo se determinó el número de especies, abundancia y diversidad de la ictiofauna de las bahías de San Agustín, Riscalillo, Chachacual, Cacaluta, Maguey, Órgano, Violín y La Entrega. La caracterización de las comunidades de peces se realizó a través de la técnica de censos visuales, se realizaron 351 transectos de 10 metros de longitud cada uno, entre enero 2009 y diciembre 2010. El análisis del número de especies, abundancia y diversidad de la ictiofauna reveló que los valores máximos fueron característicos de las bahías Maguey, La Entrega, Órgano y Violín; mientras que los valores mínimos fueron propios de San Agustín, Riscalillo, Chachacual y Cacaluta. Se detectaron diferencias significativas en la diversidad (Kruskal-Wallis $=18.71, \mathrm{P}=0.0091)$, así como en la composición y estructura de la ictiofauna entre bahías (ANOSIM=0.278; $\mathrm{P}=0.001)$. Se observó relación del número de especies de peces con el número de especies coralinas (Spearman $=0.591, \mathrm{P}<0.05)$, no siendo así para el número de especies y abundancia de peces respecto al área coralina. El número de especies coralinas y disposición de la cobertura coralina en parches, determinan hábitats de mayor complejidad estructural, influyendo en el número de especies de peces. En las localidades que poseen infraestructura turística, se observó la influencia que ejercen las actividades acuáticas recreativas sobre el comportamiento, abundancia y diversidad de la ictiofauna. La información de esta investigación puede contribuir a la integración y mejora de estrategias de manejo, conservación y protección de los recursos naturales de la región.

Palabras clave: ictiofauna, diversidad, corales, turismo, conservación, Pacífico mexicano.

\section{REFERENCIAS}

Aburto-Oropeza, O., \& Balart, F. E. (2001). Community structure of reef fishes in several habitats of a rocky reef in the Gulf of California. Marine Ecology Progress Series, 22, 283-305.

Acosta, C. A., \& Robertson, D. N. (2002). Diversity in coral reef fish communities: The effects of habitat patchiness revisited. Marine Ecology Progress Series, 227, 87-96.

Alejandre-Samaniego, D. (2010). Evaluación del grado de deterioro de la placa arrecifal de la bahía Riscalillo, Parque Nacional Huatulco (Tesis de licenciatura). Universidad Nacional Autónoma de México, México.

Allen, G. R. \& Robertson, D. R. (1998). Peces del Pacifico Oriental Tropical. México: CONABIO, agrupación Sierra Madre y CEMEX.

Almany, G. R. (2004). Does increased habitat complexity reduce predation and competition in coral reef fish assemblages? Oikos, 106, 275-284.

Álvarez-Filip, L. (2004). Influencia del Hábitat Sobre la Asociaciones de Peces en el Arrecife Coralino de Cabo Pulmo, B. C. S. Mexico (Tesis de maestría). CICIMAR-IPN, México.

Álvarez-Filip, L., Dulvy, N. K., Côté, I. M., Watkinson, A. R., \& Gill, J. A. (2011). Coral identity underpins reef complexity on Caribbean reefs. Ecological Applications, 21(6), 23-31.

Amezcua-Linares, F. (2009). Peces demersales del Pacífico de México. México: Instituto de Ciencias Marinas y Limnología, Universidad Nacional Autónoma de México.

Arias-González, J. E., Nuñez-Lara, E., Rodríguez-Zaragoza, F. A., \& Legendre, P. (2011). Indicadores del paisaje arrecifal para la conservación de la biodiversidad de los arrecifes de coral del Caribe. Ciencias Marinas, 37, 87-96.

Chabanet, P., Ralambondrainy, H., Amanieu, M., Fauré, G., \& Galzin, R. (1997). Relationships between coral reef substrata and fish. Coral Reefs, 16, 93-102.

Charbonnel, E., Serre, C., Ruitton, S., Harmelin, J. G., \& Jensen., A. (2002). Effects of increased habitat complexity on fish assemblages associated with large artificial reef units (French Mediterranean coast). ICES Journal of Marine Science, 59, 208-213.

Choat, H. J. \& Bellwood, D. R. (1991). Reef Fishes: Their History and evolution. In P. F. Sale (Ed.), The Ecology of Fishes on Coral Reef (pp. 39-47). San Diego, California: Academic Press. Inc.

Clarke, K. R. (1993). Non-parametric multivariate analysis of changes in community structure. Australian Journal of Ecology, 18, 117-143.

Clarke, K. R. \& Gorley, R. (2006). PRIMER V5: User Manual/ Tutorial. PRIMER-E Plymouth, UK. Recuperado de www.primer-e.com

Clarke, K. R., \& Warwick, R. M. (1994). Change in Marine Communities. U. K.: Plymouth Marine Laboratory. 
Comisión Nacional de Áreas Naturales Protegidas (CONANP). (2003). Programa de manejo Parque Nacional Huatulco. México, D. F.: Dirección General de Manejo para la Conservación, Comisión Nacional de Áreas Naturales Protegidas.

Di Franco, A., Baiata, P., \& Milazzo, M. (2013). Effects of recreational scuba diving on Mediterranean fishes: evidence of involuntary feeding? Mediterranean Marine Science, 14(1), 15-18.

Fonseca-Gally, S. F. (2010). Evaluación del grado de deterioro de la placa arrecifal de la bahía San Agustín, Parque Nacional Huatulco (Tesis de licenciatura). Universidad Nacional Autónoma de México, México.

Friedlander, A. M., \& Parrish, J. D. (1998). Habitat characteristics affecting fish assemblages on a Hawaiian coral reef. Journal of Experimental Marine Biology and Ecology, 224, 1-30.

Galván-Villa, C. M., Arreola-Robles, J. L., Ríos-Jara, E., \& Rodríguez-Zaragoza, F. A. (2010). Ensamblajes de peces arrecifales y su relación con el hábitat bentónico de la isla Isabel, Nayarit, México. Revista de Biología Marina y Oceanografia, 45, 311-324.

Giakoumi, S., \& Kokkoris, G. D. (2013). Effects of habitat and substrate complexity on shallow sublittoral fish assemblages in the Cyclades Archipelago, NorthEastern Mediterranean Sea. Mediterranean Marine Science, 14, 58-68.

Glynn, P. W., \& Leyte-Morales, G. E. (1997). Coral reefs of Huatulco, west Mexico: reef development in upwelling Gulf of Tehuantepec. Revista de Biología Tropical, 45, 1033-1047.

Gratwicke, B., \& Speight, M. R. (2005a). The relationship between fish species richness, abundance and habitat complexity in a range of shallow tropical marine habitats. Journal of Fish Biology, 66, 650-667.

Gratwicke, B., \& Speight, M. R. (2005b). Effects of habitat complexity on Caribbean marine fish assemblages. Marine Ecology Progress Series, 292, 301-310.

Hawkins, J. P., \& Roberts, C. M. (1993). Effects of recreational scuba diving on coral reefs: trampling on reef-flat communities. Journal of Applied Ecology, $30,25-30$.

Juárez-Hernández, L. G. (2014). Estructura de las comunidades de peces de las bahías del Parque Nacional Huatulco, con énfasis en los sistemas de arrecifes de coral (Tesis doctoral). Universidad Autónoma Metropolitana-Iztapalapa, México.

Juárez-Hernández, L. G., Tapia-García, M., \& LunaMonsiváis, B. (2013). Estructura de las comunidades de peces de las bahías Maguey y Cacaluta, Huatulco, Oaxaca. Revista Mexicana de Biodiversidad, 84(4), 1243-1257.
López-Pérez. R. A., \& Hernández-Ballesteros, L. M. (2004). Coral community structure and dynamics in the Huatulco area, Western Mexico. Bulletin of Marine Science, 75(3), 453-472.

López-Pérez, R. A., Mora-Pérez, M. G., \& Leyte-Morales, G. E. (2007). Coral (Anthozoa: Scleractinia) recruitment at Bahías de Huatulco, Western México: Implications for coral community structure and dynamics. Pacific Science, 61(3), 355-369.

López-Pérez, R. A., Calderón-Aguilera, L. E., ZepedaVilchis, R. C., López Pérez-Maldonado, I., \& LópezOrtiz, A. M. (2012). Species composition, habitat configuration and seasonal changes of coral reef fish assemblages in western Mexico. Journal of Applied Ichthyology, 29(2), 437-448.

López-Pérez, R. A., López Pérez-Maldonado, I., LópezOrtiz, A. M., Barranco Servín, L. M., BarrientosVillalobos, J., \& Leyte-Morales, G. E. (2010). Reef fishes of the Mazunte-Bahías de Huatulco reef track, Oaxaca, Mexican Pacific. Zootaxa, 2422(1), 53-62.

López-Pérez, R. A., Granja-Fernández, R., Aparicio-Cid, C., Zepeta-Vilchis, R. C., Torres-Huerta, A. M., Benítez-Villalobos, F., López-López, D. A., CruzAntonio, C., \& Valencia-Méndez, O. (2014). Corales pétreos, equinodermos y peces asociados a comunidades y arrecifes coralinos del Parque Nacional Huatulco, Pacífico sur mexicano. Revista Mexicana de Biodiversidad, 85(4), 1145-1159.

Milazzo, M., Anastasi, I., \& Willis, T. J. (2006). Recreational fish feeding affects coastal fish behaviour and increases frequency of predation on damselfish (Chromis chromis) nests. Marine Ecology Progress Series, 310, 165-172.

Monreal-Gómez, M. A., \& Salas de León, D. A. (1998). Dinámica y estructura termohalina. In M. TapiaGarcía (Ed.), El Golfo de Tehuantepec: el ecosistema $y$ sus recursos (pp. 13-26). México: Universidad Autónoma Metropolitana-Iztapalapa.

Munday, P. L. (2000). Interactions between habitat use and patterns of abundance in coral-dwelling fishes. Environmental Biology of Fishes, 58, 355-369.

Nanami, A., \& Nishihira, M. (2002). The structures and dynamics of fish communities in an Okinawan coral reef: effects of coral-based habitat structures at sites with rocky and sandy sea bottoms. Environmental Biology of Fishes, 63, 353-372.

Ramírez-Gutiérrez, J. M. (2010). Relación del hábitat con la comunidad de peces en la bahía San Agustín. Huatulco, México (Tesis de Maestría). Universidad Nacional Autónoma de México, México.

Ramírez-Gutiérrez, J. M., Tapia-García, M., Ramos-Santiago, E., \& Ulloa, R. (2007). Fish community structure in San Agustín Bay, Huatulco, Mexico. Revista Chilena de Historia Natural, 80(4), 419-430. 
Sale, P. F., \& Douglas, W. A. (1981). Precision and accuracy of visual census technique for fish assemblages on coral patch reefs. Environmental Biology of Fishes, 6(3-4), 333-339.

Sale, F. P., \& Douglas, W. A. (1984). Temporal variability in the community structure of fish on coral patch reef and the relation of community structure to reef structure. Ecology, 65(2), 409-422.

Sale, P. F., \& Dybdahl, R. (1975). Determinants of community structure for coral reef fishes at settlement. Ecology, 56, 1343-1355.

Shannon, C. E., \& Wiener, W. (1963). The mathematical theory of comunication. Illinois: University of Illinois.
Tapia-García, M., Stender, K., \& Juárez-Hernández, L. G. (2007). Guía de identificación de los corales pétreos de las Bahías de Huatulco. Colección "Manuales de Identidad de la CONANP". México: CONANP-SEMARNAT.

Tokeshi, M., \& Arakaki, S. (2012). Habitat complexity in aquatic systems: fractals and beyond. Hydrobiologia, $685,27-47$.

Zakai, D., Levy, O., \& Chadwick-Furman, N. E. (2000). Experimental fragmentation reduces sexual reproductive output by the reef building coral Pocillopora damicornis. Coral Reefs, 19, 185-188. 